\title{
Establishing a community-based smoke-free homes movement in Indonesia
}

\author{
Retna Siwi Padmawati ${ }^{1,2}$, Yayi Suryo Prabandari ${ }^{1,2}$, Tutik Istiyani ${ }^{2}$, Mark Nichter $^{3}$, Mimi Nichter $^{3}$
}

\begin{abstract}
INTRODUCTION Indonesia has one of the highest male smoking rates in the world (67\%) and secondhand smoke (SHS) exposure occurs in over $70 \%$ of households. To date, little research has investigated community recognition of the harms of secondhand smoke and support for a smoke-free homes (SFH) policy. This work discusses the development and implementation of a community-based SFH intervention attempting to establish SFH as a new social norm.

METHODS Research was conducted in Yogyakarta, Java. A proof-of-concept study ascertained the feasibility of mounting a community-based SFH initiative in urban neighborhoods. Educational materials on SHS were developed and pretested. An intervention was piloted and evaluated in the homes of 296 smokers residing in 4 communities. Health educators and community health volunteers were trained to implement SFH.

RESULTS Prior to the intervention, $11 \%$ of smokers did not smoke inside their home; post-intervention $54 \%$ of smokers did not smoke inside their home. The Yogyakarta District Health Office has supported large scale implementation of smoke-free homes. To date, 135 urban communities have declared themselves as having SFH. CONCLUSIONS This is the first community-based SFH initiative to be carried out in South-East Asia. The SFH movement redefines smoking cessation as a health issue of women and children, ties family welfare to core cultural values, and offers women a leadership role in tobacco control. The sustainability of SFH in Yogyakarta has been achieved by working closely with multiple levels of government and has contributed to shifts in tobacco control policy in Indonesia.
\end{abstract}

\begin{abstract}
AFFILIATION
1 Department of Health Behavior, Environment and Social Medicine, Faculty of Medicine, Public Health and Nursing, Universitas Gadjah Mada, Yogyakarta, Indonesia 2 Center for Health Behavior and Promotion, Faculty of Medicine, Public Health and Nursing, Universitas Gadjah Mada, Yogyakarta, Indonesia 3 School of Anthropology, University of Arizona, Tucson, Arizona, United States

CORRESPONDENCE TO Mimi Nichter. School of Anthropology, University of Arizona, Emil Haury Building, 85721 Tucson, Arizona, United States. E-mail: mimi.nichter@ gmail.com
\end{abstract}

\section{KEYWORDS}

Indonesia, secondhand smoke, global health, smoke-free homes

Received: 25 February 2018 Revised: 22 October 2018 Accepted: 1 November 2018

\section{INTRODUCTION}

Smoking is a pressing public health concern in Indonesia. The country ranks as the fourth largest consumer and the fifth largest producer of tobacco in the world, and cigarette prices are among the lowest globally ${ }^{1}$. National survey data reveal that $67 \%$ of men and $4 \%$ of women are current smokers ${ }^{2}$. Cigarette consumption is on the rise among both adults and children. Between 2001 and 2010, the number of children, between the ages of 10 and 14 years, who started smoking rose by $80 \%^{3}$.
To date, the government has moved slowly toward implementation of tobacco control policies and tobacco continues to be aggressively marketed in a largely unrestricted environment ${ }^{4,5}$. In 2009 , the Ministry of Health passed legislation that all provinces and districts in the country should pass their own smoke-free regulations, but to date only half of the districts have done so. Indonesia is the only country in the Asia-Pacific region that has not ratified the WHO Framework Convention on Tobacco Control. 
The prevalence of secondhand smoke (SHS) exposure among non-smokers in Indonesian homes is $72 \%^{2}$. The dangers of SHS are not widely known in the country and are not commonly taught in medical schools $^{6}$. Scientific evidence has long concluded that there is no safe level of SHS exposure and that exposure leads to cardiovascular and respiratory diseases as well as lung and other cancers ${ }^{7}$.

Reducing women and children's exposure to SHS in the home is an important public health issue $\mathrm{e}^{8,9}$. Interventions to create smoke-free homes ( $\mathrm{SFH}$ ) have been developed in many countries and have met with varying success ${ }^{10,11}$. Some interventions in high-income countries have shown that a smokefree home can be an effective aid in reducing the daily consumption level of both heavier and lighter smokers and can increase the likelihood of quitting ${ }^{12,13}$. Whereas previous efforts to develop $\mathrm{SFH}$ have largely focused on shifting individual behavior, the $\mathrm{SFH}$ movement described in this paper had the goal of changing community-wide smoking norms inside homes. We draw upon social practice theory and research that has documented ways in which community social norms (defined as widely adhered to rules that dictate acceptable, if not desired conduct) impact on smoking practices ${ }^{14}$ as enactments of larger cultural conventions ${ }^{15}$. The SFH movement sought to change smoking norms in central Javanese neighborhoods marked by a high degree of collective efficacy ${ }^{16}$, given strong local leadership and group cohesiveness. To do so, the harms of SHS to women and children needed to become widely recognized, and positive cultural meanings needed to be associated with non-smoking practices in homes.

In Indonesia, little is known about household and community attitudes toward SHS or social support for $\mathrm{SFH}^{17,18}$. A survey was conducted by our research team in 2010 to ascertain the extent to which women and children were exposed to SHS in their homes and attitudes toward an SFH initiative ${ }^{18}$. While most women (77\%) did not think that they could introduce a smoke-free rule in their home as an individual act, $85 \%$ per cent of women expressed enthusiasm about introducing $\mathrm{SFH}$ as a community norm, and many men, even smokers, reported that they would endorse it. While we recognized the importance of tobacco cessation, we determined through formative research that the $\mathrm{SFH}$ initiative would have a greater chance of success if the focus was not on quitting but rather on not smoking inside the home. The unit of analysis of this intervention was smoking cessation within the home as a step contributing to smoking cessation in general ${ }^{19}$.

In this paper, we discuss action research conducted by Project Quit Tobacco International (QTI) towards an end to developing, refining and implementing an SFH intervention and then bringing it to scale as a social movement. QTI is a smoking cessation research collaborative working in Indonesia and engaged in clinic and communitybased tobacco control activities ${ }^{19}$.

\section{METHODS}

Smoke-free home interventions were developed and implemented in Yogyakarta (2010-2018), a city of about half a million people located in the southern part of Central Java. Ethical clearance for conducting research and $\mathrm{SFH}$ interventions was obtained from Universitas Gadjah Mada, Faculty of Medicine.

\section{Stage One: Proof-of-concept}

The QTI team conducted a proof-of-concept study to ascertain the feasibility of mounting a communitybased SFH initiative in urban neighborhoods (kampung), comprising approximately 200 households. Communities selected for participation in Stage One had a neighborhood association with strong leadership and an active women's group (known locally as Pembinaan Kesejahateraan Keluarga, or PKK, translated as the Family Welfare Movement). Our rationale for working with the PKK was that it has a history of participation in government health campaigns. Its members already have training in hygiene and nutrition, are seen as promoters of community health, and many function as community health volunteers (kaders). Partnering with a strong and well-established community health movement in Central Java was a means to establish legitimacy for the SFH initiative.

We first approached community and women's group leaders to raise their awareness about the harms of SHS, gauge their interest in implementing an SFH policy in their community, and to obtain ideas about how this could best be done. Meetings were audio-recorded, transcribed, and translated 
into English from Bahasa Indonesia. Members of the team reviewed the transcripts, noting what facts about SHS were deemed salient by community leaders, suggestions about how an SFH intervention might be implemented, and challenges they foresaw.

Six focus group discussions (FGDs) were conducted with women $(n=48)$ whose husbands smoked inside the home and two FGDs were conducted with male smokers $(n=15)$. FGDs explored a range of issues including household rules about smoking, knowledge about the harm of SHS, and willingness to participate in an SFH initiative (Table 1).

\section{Stage Two: Developing educational materials}

A visual presentation was developed on the harms of SHS to women and children and the relationship of smoking to a wide range of health problems. Three groups of community members were shown the presentation in order to obtain their feedback. Questions and concerns raised about the harms of SHS were incorporated into educational questionand-answer brochures that explained in lay terms the harms of smoking inside the home. A brochure on how to quit smoking was also developed. The QTI team also created a variety of stickers, posters and banners, to post outside homes and around the community, to indicate to community members and visitors that an SFH policy was being implemented. Development of the visual and printed materials was an iterative process wherein materials were pretested by QTI staff with community members and refined based on feedback.

Following a suggestion from community leaders, an educational video was developed on the harms of SHS, featuring prominent doctors and laying out the steps entailed in becoming an SFH community.

The video was pretested with community members and feedback was incorporated in a final edit. The video was envisioned as a tool to facilitate educational sessions in communities and to enable standardizing and streamlining the process of $\mathrm{SFH}$ implementation so that the program could be scaled up with ease.

\section{Stage Three: Piloting the intervention}

In Stage Three, QTI staff in conjunction with the District Health Office, selected 4 communities with
Table 1. Steps in the process of becoming a smoke-free homes community

\section{Steps} Activities

Step 1

- Organize a meeting of community leaders and heads of women's groups.

- Present data on harms of secondhand smoke.

- Explain rationale for establishing smoke-free homes in their community.

- If group expresses interest, ask for their assistance in arranging an educational meeting for community members.

Step 2

- Arrange 3 large scale educational meetings where Primary Health Center staff provide facts about harms of SHS and importance of becoming a smoke-free homes community.

- Encourage women and men to attend to garner widespread support.

- Show SFH video featuring prominent doctors and the testimonials of other communities that have adopted a smoke-free homes policy. Follow this with a question \&t answer session about the harm of SHS to the family facilitated by a local doctor.

- Emphasize that the initiative is not asking men to quit smoking but rather not to smoke inside their house.

- Encourage men who are smokers to participate in establishing a new community norm.

Step 3

- Community health volunteers (kaders) who have been trained in harms of SHS and community mobilization work to familiarize all households with the SFH initiative in order to gain consensus for a community wide SFH declaration.

- Kaders talk with women whose husbands are resistant to the idea of SFH and provide positive reasons for participation in the movement.

- SFH stickers are distributed to households by kaders to acknowledge that the home is smoke-free.

Step 4

- Arrange for and hold a smoke-free homes declaration meeting for all community members.

- Agreement is reached on the actions and activities to initiate and enforce a smoke-free home policy. The declaration should include the following: 1) no smoking should occur in homes for both household members and guests, 2) no smoking is allowed at community meetings, 3) no smoking in the home stickers are to be placed on the front door of all households in the community, and 4) no smoking is allowed in front of children and pregnant women even outside the home. Communities may choose to add other points to their declaration.

- Prominent health officials are invited to make it clear that this is an action of significance.

- Speeches are given and the components of the smokefree homes initiative are read to all gathered and clarified.

- Media are invited to garner publicity for the event.

- Declaration is signed by important leaders of the community and health officials. Following signing, declaration is placed in a prominent place in the community. 
a history of successful health outreach activities in which to pilot the intervention. The objective was to see how SFH could be introduced under ideal conditions by well-trained QTI staff, dedicated community leaders and motivated community health volunteers, kaders.

The pilot interventions included 3-6 educational sessions in each community that were conducted by QTI staff with a Primary Health Center (PHC) doctor or health educator also in attendance. The video was shown, followed by a question-and-answer session. A few weeks after these sessions, a large communitywide event was planned by community leaders and the PKK, at which time the SFH intervention was officially launched. Banners and posters about the SFH initiative were placed in key locations, and SFH stickers were distributed to each household by the kaders. QTI staff had frequent phone contact with kaders in the months following the event, enquiring about the success and challenges of the $\mathrm{SFH}$ initiative in their community.

Twelve months after the intervention was piloted in these communities (2014), a survey was administered amongst households $(n=296)$, where the husband smoked, to assess changes in smoking inside the home and knowledge of the harms of SHS. The instrument was pretested in the community and modified to enhance comprehension. In 2018, phone interviews were conducted by the QTI team with 10 kaders working in the communities to understand whether the smoke-free norm had been maintained by male household members and guests.

\section{Stage Four: Piloting by the District Health Office}

Two communities were selected by the District Health Office to test the capacity of government health educators from PHC and community-based kaders to implement and monitor the SFH program without direct QTI staff assistance. To this end, two health educators and six kaders were trained by QTI researchers on: how to discuss the harms of SHS with community members; how to conduct interactive sessions using the educational video; and how to assist communities implement an SFH policy.

Health educators and kaders introduced the intervention in the community following the same process as QTI staff and were provided all relevant materials. QTI staff observed their meetings, attended the community-wide launch meeting of the SFH initiative, and had phone contact with the health staff to monitor how the program was unfolding.

\section{Stage Five: Going to scale}

In Stage Five, the District Health Office adopted the SFH program and funded its large-scale implementation. This entailed QTI training two health educators from each of the 18 PHCs in the district. Following training, health educators at each PHC selected two or three communities in which to introduce the intervention that were to serve as exemplars for other communities. Kaders from the communities were trained by QTI staff.

\section{Data collection and analysis}

Both qualitative and quantitative data were collected. During the formative stage, FGDs were conducted to better understand smoking practices inside the home and to guide intervention development. FGDs were audio-recorded, transcribed, and translated into English from Bahasa Indonesia. Key themes were identified by team members and a codebook was developed. Coding assisted in the organization of the data, and new themes were added as they emerged. Transcripts were individually coded by three teammembers and then reviewed as a team. Discrepancies in coding and the meaning of Indonesian terms were discussed and resolved during team meetings.

Observational data and ongoing discussions with community leaders, health educators, and kaders were used to develop, implement, and monitor the intervention. Ongoing data collection gathered from phone interviews with kaders enabled tracking of the success and challenges of the intervention. Postintervention, survey data $(n=296)$ were entered and analyzed with the Statistical Package for the Social Sciences (SPSS) Version 21.0. Categorical variables were presented as frequencies and percentages and compared using chi-squared tests to determine whether smoking behaviors in the home differed pre- and post-intervention.

\section{RESULTS}

Stage One: Proof-of-concept

Findings from focus groups

Four key themes emerged from the analysis of focus 
group data. First, although most women disliked their husband's smoking inside the home, many felt powerless to ask him not to smoke as this was considered culturally inappropriate and a direct challenge to a man's authority. Women expressed fear that doing so might provoke anger from their husband. The QTI team were concerned that angering the husband by asking him not to smoke in the house could result in violence against the wife. It was clear that developing non-confrontational ways of introducing $\mathrm{SFH}$ to male smokers would be a critical issue in the success and safety of the intervention.

Second, few households had rules about not smoking in the home, even in cases where a woman was pregnant or there was a baby in the house. A highly sensitive issue was requesting that guests not smoke inside the house. This was particularly inappropriate when an elder person was smoking. Women explained that they could not embarrass any guest directly by asking them not to smoke. To do so was deemed impolite given that smoking is a normative part of Indonesian male culture. When asked about posting 'this is a smoke-free home' signage on the door, women noted that this might be effective as an indirect communication strategy.

A third emergent theme was that women had little sense of self-efficacy in being able to convince their husband not to smoke inside the home. We queried ways of fostering collective efficacy given the importance of social norms in Javanese culture. When we asked whether SFH could be introduced as a community-wide norm, women expressed enthusiasm about this possibility and noted that their husbands might be receptive to such an approach, if introduced by community leaders.

A fourth theme was a lack of awareness that SHS was harmful to health. Secondhand smoke was viewed by women as more of an annoyance than a health hazard. Among men, we found that there was little knowledge that SHS was harmful for women and children. Both men and women expressed great interest in learning more about children's illnesses caused by SHS.

A fifth theme that arose in FGDs with men was the positive response to introducing an SFH policy in their community as an act of male responsibility for women and children's health. Men stated that the SFH movement would be supported if it was endorsed by the entire community and if they could continue smoking outside the home.

\section{Insights from community leaders}

Discussions with community leaders provided further insight into possible outcomes of an $\mathrm{SFH}$ intervention. As one leader explained: 'If there is a social movement to stop smoking inside the home, people in our community will follow. Now men are afraid to turn down a cigarette-if you are offered one you must accept or others will ask "why are you not smoking?". The only acceptable reason for turning. a cigarette down is that you are feeling ill. For us Javanese men, it is very important to do things that others are doing.... so if there is a smoke-free homes movement, others will join.'.

Community leaders also noted that the presence of doctors and health officials at SFH activities would establish credibility for the movement and would result in increased support for the initiative from community members. It was further suggested that a formal printed declaration be developed and signed by key community leaders stating that the community supported an SFH policy (Table 1). They suggested that the signing of this declaration should take place at a large community event, and that after signing the declaration it should be hung in the main community hall to serve as a visual reminder to community members of their decision to make homes and meetings smoke-free.

\section{Stage Two: Community response to educational materials}

Educating community members about the harms of SHS was a critical first step in gaining their participation. To do so, we generated educational materials that appealed to the public by addressing the relationship of SHS to the kinds of health problems people were most interested in learning about and highlighting facts about SHS that best captured their attention. From FGDs, QTI researchers learned that community members were interested in childhood illnesses related to SHS (i.e. respiratory illness, asthma), heart disease and diabetes. They responded positively to pictures of 'people suffering from terrifying diseases' 
that helped visualize the dangers of smoking (e.g. blackened lungs; amputations as a result of gangrene).

Participants in preliminary educational meetings were surprised to learn that SHS was as harmful to health as smoking and that smoking in one room of the house (a common practice) was ineffective in reducing SHS exposure in the rest of the house. One challenge noted by kaders was convincing community members that smoke particles remained in the air even after people could not see smoke. In response, QTI experimented with using a smoke detection device to demonstrate that smoke remained in the air far longer than community members imagined. Although the numerical readout from the device was not understood, the technology had symbolic value and proved effective. Footage of using the device in a home was added to the educational video as a reminder to community members that smoke residue posed a danger to all household members. A final version of the video is available on the QTI website ${ }^{20}$.

\section{Stage Three: Piloting the intervention}

We piloted the intervention in four communities. In each community, three well-attended educational meetings were held. The video was well received by those in attendance. Following these events, a declaration meeting was conducted in each of the four communities. Declaration meetings were typically attended by 200-400 people including community members, local leaders and dignitaries, and health staff from the local, district and provincial levels. QTI staff arranged for press coverage at the declaration event, as community leaders expressed an interest in being identified at the vanguard of the SFH movement. Social recognition of the declaration event was found to be an important motivator enhancing community participation in the $\mathrm{SFH}$ initiative. Community leaders also saw the event as an opportunity to encourage attendees to participate more broadly in 'healthy lifestyles' so before the event began, they sponsored a walking/cycling event around the community.

Smoke-free homes signage was found to be essential in establishing the home as an offlimits space for smoking. Signage was embraced by women as a means to convey the smoke-free message to guests in a non-confrontational manner in keeping with Javanese culture that values indirect communication. Women often pointed to the SFH sticker rather than overtly asking a person in their house not to smoke and requested that the sticker size be enlarged so guests could more easily read the no-smoking message.

Kaders played an important role in implementing and monitoring $\mathrm{SFH}$ activities and expressed commitment to changing household smoking norms. They explained that having printed materials on SHS to hand out to households legitimated their role in promoting SFH policy.

\section{Follow-up surveys and phone interviews in pilot} communities

Twelve months after the declaration meetings (2015), a retrospective survey was conducted in the four pilot kampung to ascertain changes in knowledge about SHS and the extent to which the smoke-free policy had been adopted. Prior to the intervention, it had been established through formative research that the local population had little awareness that SHS could cause disease ${ }^{18}$. In the retrospective survey, when asked if SHS caused illnesses, $89 \%$ of respondents noted that it could cause serious illness, $6 \%$ responded that it could cause mild illness, and $5 \%$ were not sure. Table 2 presents data on which diseases were associated with SHS, after the intervention.

Notably, community members were now well

Table 2. Follow up survey results: Knowledge of illnesses associated with secondhand smoke

\begin{tabular}{lcc} 
& Frequency & Percentage \\
Illiness & 228 & $(\%)$ \\
\hline Low birthweight baby* & 213 & 77 \\
Children's asthma & 287 & 97 \\
Children's respiratory illness & 219 & 74 \\
Adult asthma & 290 & 98 \\
Adult respiratory illness & 228 & 77 \\
TB & 278 & 94 \\
Adult lung cancer* & 260 & 88 \\
CVD* & 181 & 61 \\
Stroke & 47 & 16 \\
Diabetes complications &
\end{tabular}


aware that SHS could cause low birth weight in babies as well as respiratory and heart disease. Less well recognized was that SHS could place one at risk to diabetes and lead to complications.

Of particular importance to the SFH initiative was whether husbands reduced or stopped smoking inside their home. Pre-intervention, only $11 \%(n=30)$ of the smokers did not smoke inside the home while $89 \%(n=266)$ did. Post-intervention, 54\% $(n=161)$ of smokers were no longer smoking inside their home while $46 \%(n=135)$ of men did. This reduction in household smoking is statistically significant $(\mathrm{p}<0.01)$. With regard to frequency, pre-intervention, $52 \%(n=138)$ of men who smoked in the house did it frequently and $48 \%(n=128)$ did it rarely $(<5$ times a month). Post-intervention, among those who continued to smoke inside the home, $55 \%(\mathrm{n}=74)$ smoked frequently and $45 \%(\mathrm{n}=61)$ smoked rarely.

There is evidence that the smoke-free norm introduced in the four kampung has been sustained. Telephone interviews conducted in 2018 with ten kaders serving the four pilot communities found that between $70-90 \%$ of households that they routinely visited were still adhering to the SFH policy. Kaders also noted that women had become more assertive in communicating with their husband about not smoking inside the house as a result of the shift in social norms that had occurred due to the SFH initiative. The signed declaration, nosmoking stickers displayed on every household in the neighborhood, as well as the banners and posters around the community, further contributed to women's assertiveness. In effect, women could now speak with the backing of the community (collective efficacy), rather than making an individual request to male smokers. Kaders also noted that guests visiting from outside the community were compliant because of signage. They further noted that men felt embarrassed when seen by others smoking inside their homes.

\section{Stage Four: Piloting by District Health Office}

After it was demonstrated that QTI staff working with kaders could mount an effective SFH campaign, the question arose whether PHC health educators could do so, assisted by kaders. Two communities were selected by the District Health Office for a pilot study. Health educators were trained in how to conduct SFH outreach programs using the protocol and materials developed by QTI. QTI staff observed that health educators were comfortable presenting the educational video, but often required assistance in answering questions from community members. For example, in the question-and-answer session, community members wanted to know if the toxins of SHS could be removed from the body and how long it would take to do so. QTI staff developed culturally appropriate responses to these types of questions to be incorporated in future training for health educators.

\section{Stage Five: Going to scale}

Based on the success of the pilot studies, SFH has gone to scale supported by both the Yogyakarta Provincial and District Health Offices. To date, health educators from all the PHCs in Yogyakarta City $(n=36)$ have been trained by QTI staff to conduct educational sessions on SHS and additional kaders have been trained to work in the communities. One hundred and thirty-five kampung (out of 600 in Yogyakarta) have thus far become smoke-free, and the movement continues to grow. The District Health Office continues to encourage PHCs to be proactive in selecting communities for $\mathrm{SFH}$ implementation, and communities are themselves requesting SFH programs even when not actively recruited. Experienced health educators are now functioning as trainer of trainers in the smoke-free movement with QTI staff serving as a resource when requested.

Healthy competition between communities has helped sustain interest in SFH. Competition is promoted by the District Health Office in the form of prizes and various forms of public recognition. To gain recognition, many communities customized their SFH activities and added something unique not featured in the SFH video, such as decorating flower pots to serve as ashtrays placed in front of each household, including SFH messages in school-based programs, and sponsoring other healthy lifestyle events. Competition contributed to sustainability through ongoing innovation, reminding community members of their commitment to SFH policy.

\section{DISCUSSION}

The prime objective of the intervention was to promote a community-based smoke-free home 
norm. In order to do so, the SFH intervention needed community leaders' support and needed to be implemented by motivated health staff and kaders. An outcome evaluation of pilot communities found that a critical mass of homes was adhering to the SFH policy. Prior to the intervention, $11 \%$ of smokers did not smoke inside their home; post-intervention $54 \%$ of smokers were no longer smoking inside their home. Recent phone interviews with ten kaders confirmed that motivation for the $\mathrm{SFH}$ initiative continues to be high in the households they monitor and has been sustained at about a 70\% level.

It is worth considering the cultural and administrative context of SFH success in Yogyakarta and factors likely to influence its sustainability. There are two aspects of Javanese culture that both favor and challenge community-based tobacco control efforts in central Java. On the positive side are the strong communal values of collective action ${ }^{21}$. These aspects of kampung life have been well documented $^{21,22}$ and are readily visible in collective praying and the many meetings, communal work events, rotating credit groups, rituals, and social media networks found in each community. SFH was carefully introduced to build on these aspects of Javanese culture. The SFH movement is nonconfrontational. Formative research in Yogyakarta found that strong anti-smoking messages would likely undermine SFH efforts as a tobacco control foothold in communities. It needs to be remembered that the SFH initiative was launched at a time when awareness of the harms of smoking in Indonesia was low ${ }^{18,19}$. SFH was designed to be a first step in tobacco control through building consensus around an SFH norm endorsed by all residents.

A second aspect of Javanese culture presents a challenge to tobacco control efforts. Social exchange, in the form of commensality and accepting any food or food-drug ${ }^{23}$ offered as an act of friendship or inclusion, is a strong social norm. Cigarettes are commonly exchanged as an inexpensive commodity. At baseline, cigarettes were often handed out during kampung meetings, community-based work events, funerals, etc. The tobacco industry has exploited smoking as a valued social activity in its advertisements and has made it appear that smoking is closely tied to cultural values in Indonesia ${ }^{4}$. In order for SFH to be seen as culturally acceptable it had to be introduced in a way that played up cultural values that superseded the offering and exchange of cigarettes as a social good. The cultural value promoted by SFH was male responsibility for the welfare of one's family.

Politically, Indonesia has moved from an era of strong central control to decentralized rule, which has had the effect of shifting decision-making power from provinces to districts and smaller administrative units $^{24,25}$. This effected SFH implementation as budgets for promotive health activities are now available at each tier. In order to be sustained, $\mathrm{SFH}$ had to secure sufficient funds from multiple government stakeholders. SFH was able to receive financial support for activities like declaration meetings and training events from stakeholders at the Provincial, District, and Primary Health Centers.

Policy advocated by the national government has also influenced and been influenced by SFH. In 2011, the Ministry of Health began vigorously promoting a healthy lifestyle agenda that has been passed down to provincial and district health offices $^{26}$. These offices have in turn promoted healthy lifestyle activities at the level of the kampung to meet national indicators of excellence. Reducing the number of smokers in households was originally a healthy lifestyle indicator. In part, due to QTI advocacy and the success of the SFH movement in Yogyakarta, the national indicator has now been changed to smoke-free households. To achieve ongoing measures of success, District Health Offices in Yogyakarta continue to be proactive in encouraging $\mathrm{PHCs}$ to promote $\mathrm{SFH}$ programs in new kampung each year. This contributes to the sustainability of the SFH movement.

\section{Limitations}

Two limitations are noted. First, the SFH intervention was conducted in Java, and findings may not be generalizable to other parts of the Indonesian archipelago. Second, the retrospective survey to assess effectiveness of the SFH initiative was conducted twelve months post-intervention. Recall bias is possible as women were asked to report on their husband's smoking behavior one year in the past. To date, only four pilot communities have been evaluated for success of the program, and these were subject to these limitations. 


\section{CONCLUSIONS}

In this work, we have described the development and implementation of a community-based $\mathrm{SFH}$ movement in Indonesia. To our knowledge, this is the first community-based SFH movement to be carried out in South-East Asia. Notably, the SFH movement reframes smoking cessation as a health issue of women and children, which ties their welfare to core cultural values indexing male responsibility. In so doing, the smoke-free homes initiative has offered women an important leadership role in tobacco control as well as contributing to a tobacco control harm reduction agenda ${ }^{27}$. This study has also called attention to the importance of integrating $\mathrm{SFH}$ into larger healthy lifestyle programs increasingly promoted by the Government of Indonesia. The success and sustainability of SFH in Yogyakarta has depended upon working closely with stakeholders at multiple levels of government. By doing so, it has contributed in important ways to changing local and national tobacco control policy in Indonesia.

\section{REFERENCES}

1. Eriksen M, Mackay J, Ross H. The tobacco atlas. Atlanta, GA: American Cancer Society. New York: World Lung Foundation; 2014.

2. World Health Organization. Global adult tobacco survey: Indonesia report 2011. New Delhi: World Health Organization Regional Office for South East Asia; 2012.

3. Center for Data and Information Ministry of Health. Smoking behavior in Indonesian communities. http:// www.depkes.go.id/folder/view/01/structure-publikasipusdatin-info-datin.html. Published May, 2015. Accessed February 23, 2018.

4. Nichter M, Padmawati RS, Danardono M, Ng N, Prabandari Y, Nichter M. Reading culture from tobacco advertisements in Indonesia. Tobacco Control. 2009;1(April):18(2):98-107. doi: 10.1136/tc.2008.025809

5. Rosser A. Contesting tobacco-control policy in Indonesia. Critical Asian Studies. 2015. 47(1). pp. 6993(25). doi: 10.1080/14672715.2015.997083

6. Prabandari YS, Nichter M, Nichter M, Padmawati RS, Muramoto M. Laying the groundwork for tobacco cessation education in medical colleges in Indonesia. Education for Health. 2015;1(September):28(3):169. doi: $10.4103 / 1357-6283.178602$

7. World Health Organization. Report on global tobacco epidemiology 2017: Monitoring tobacco use and prevention policies. Geneva: World Health Organization; 2017.

8. Bloch M, Althabe F, Onyamboko M, Kaseba-Sata C, Castilla EE, Freire S, et al. Tobacco use and secondhand smoke exposure during pregnancy: An investigative survey of women in 9 developing nations. American J. Public Health. 2008;9(October):1833-40. doi: 10.2105/AJPH.2007.117887

9. Wipfli HL, Samet JM. Second-hand smoke's worldwide disease toll. The Lancet. 2010;26 (November):377(9760):101-2. doi: 10.1016/S0140-6736(10)61922-8

10. Baxter S, Blank L, Everson-Hock ES, Burrows J, Messina J, Guillaume L, et al. The effectiveness of interventions to establish smoke-free homes in pregnancy and in the neonatal period: A systematic review. Health Ed. Research. 2011;26(January):265-82. doi: 10.1093/her/cyq092

11. Alwan N, Siddiqi K, Thomson H, Lane J, Cameron I. Can a community-based 'smoke-free homes' intervention persuade families to apply smoking restrictions at homes?. J of Public Health. 2010;7(October):33:48-54. doi: 10.1093/pubmed/fdq073

12. Mills AL, Messer K, Gilpin EA, Pierce JP. The effect of smoke-free homes on adult smoking behavior: A review. Nicotine \& Tobacco Research 2009;24(July):11(10):11311141. doi: $10.1093 / \mathrm{ntr} / \mathrm{ntp} 122$

13. Hyland A, Higbee C, Travers MJ, et al. Smoke-free homes and smoking cessation and relapse in a longitudinal population of adults. Nicotine \& Tobacco Research. 2009;3(April):11(6):614-618. doi: 10.1093/ntr/ntp022

14. Karasek D, Ahern J, Galea S. Social norms, collective efficacy, and smoking cessation in urban neighborhoods. American J. Public Health. 2012;102:343-351. doi:10.2105/AJPH.2011.300364

15. Blue S, Shove E, Carmona C, Kelly, MP. Theories of practice and public health: Understanding (un) healthy practices. Crit Pub Health. 2016:26 (1):36-50. doi: 10.1080/09581596.2014.980396

16. Bandura A. Exercise of human agency through collective efficacy. Current Directions Psychological Science. 2000;9(3):75-78. doi:10.1111/1467-8721.00064

17. Kaufman MR, Merritt AP, Rimbatmaja R, Cohen JE. 'Excuse me, sir. Please don't smoke here'. A qualitative study of social enforcement of smokefree policies in Indonesia. Health Policy and Planning. 2015;22(September):30(8):995-1002. doi: 10.1093/heapol/czu103

18. Nichter M, Nichter M, Padmawati RS, Ng N. Developing a smoke free household initiative: An Indonesian case study. Acta Obstetricia et Gynecologica Scandinavica. 2010;(April):89(4):578-81. doi: 10.3109/00016340903578893

19. Nichter M, Nichter M, Muramoto M, Project Quit Tobacco International. Project quit tobacco international: Laying the groundwork for tobacco cessation in low-and middle-income countries. Asia Pac. J. Public Health. 2010;(July):22,3 181S-8S. doi: $10.1177 / 1010539510373023$

20. Project Quit Tobacco International. http://www. quittobaccointernational.org. Published February 2018. 
Accessed February 21, 2018.

21. Guinness P. Kampung, Islam and state in urban Java. Singapore: NUS Press National University of Singapore; 2009.

22. Newberry J. Rituals of rule in the administered community: The Javanese slametan reconsidered. Modern Asian Studies. 2007;(November):41(6):1295-329. doi: 10.1017/S0026749X06002575

23. Jankowiak WR, Bradburd D, eds. Drugs, labor, and colonial expansion. University of Arizona Press; 2003.

24. Heywood P., Choi Y. Health System Performance at the District Level in Indonesia after Decentralization. BMC International Health and Human Rights. 2010:10(3):112. doi:10.1186/1472-698X-10-3

25. Aspinall E. Health Care and Democratization in Indonesia. Democratization. 2014: 21(5):803-823. doi:10.1080/13510347.2013.873791

26. Ningsih FG, Jonyanis S. Clean and Health Living Behaviors in Gunung Kesiangan community. JOM FISIP. 2014;1(2):1-5.

27. Amos A, Greaves L, Nichter M, Bloch M. Women and tobacco: A call for including gender in tobacco control research, policy and practice. Tobacco Control. 2011;21(2):236-243. doi: 10.1136/tobaccocontrol-2011-050280

ACKNOWLEDGEMENTS

The authors thank the QTI Indonesia Research Team: Jusniar Dwi Rahaju, Didik Joko Nugroho, Endang Pujiastuti, and Mahesto Danardono; the Yogyakarta Health Office, the health educators of the Primary Health Centers, and all the kaders who participated in the smoke-free homes intervention.

CONFLICTS OF INTEREST All authors report (ICMJE form disclosure) a grant from Fogarty International Center, National Institutes of Health, USA, during the conduct of the study. The authors declare that they have no competing interests, financial or otherwise, related to the current work.

FUNDING

This work was supported by the Fogarty International Center of the National Institutes of Health, USA, under award number R01TW007944. The content is solely the responsibility of the authors and does not necessarily represent the official views of the NIH.

PROVENANCE AND PEER REVIEW

Not commissioned; externally peer reviewed. 